\title{
Dissociated unilateral convergence paralysis in a patient with thalamotectal haemorrhage
}

\author{
K Lindner, P Hitzenberger, M Drlicek, W Grisold
}

\begin{abstract}
A 47 year old male was admitted in a comatose state. CT scan showed a haemorrhage in the right pulvinar thalamus descending into the right part of the lamina quadrigemina. He presented with anisocoria, prompt bilateral pupillary light reaction, and unilateral convergence paralysis contralateral to the lesion in combination with upward gaze palsy. During an observation period of two months, the convergence reaction returned to normal. MRI showed a lacunar lesion ventral to superior right colliculus. Angiography revealed an arteriovenous malformation (right posterior cerebral artery-sinus rectus) as the possible cause of the haemorrhage.
\end{abstract}

(F Neurol Neurosurg Psychiatry 1992;55:731-733)

The convergence reaction is a complex function consisting of pupillary constriction, accommodation and adduction of both eyes. Abnormalities of convergence have been described in congenital defects and a number of neurological disorders. ${ }^{1}$ In acquired disease, impairment of convergence is often combined with abnormalities of vertical gaze as observed in Parinaud's syndrome, ${ }^{2}$ Parkinsonism, ${ }^{34}$ progressive supranuclear palsy, ${ }^{5}$ tumours of the pineal corpus ${ }^{56}$ or dorsal midbrain infarctions. ${ }^{6}$ Neurological diseases with intact pupillary light reaction and absent pupillary convergence reaction suggest that pathways for pupillary light and near reaction are different.

\section{Case report}

A 47 year old hypertensive man was admitted in a comatose state. Upon recovering from coma, the patient was quadruparetic and had right sided ataxia.

Ophthalmoneurologically the eyelids were symmetric; pupils were round but anisocoric, the left pupil was larger than the right one. Pupillary light reaction (direct and indirect) was bilaterally prompt. In the primary position, the right eye was normally positioned, the left eye was slightly adducted and downwardly positioned. Vertical upward gaze palsy was noted. When attempting to look down, in addition to the downward gaze, a mild symmetric adduction of both eyes without nystagmus occurred. Horizontal gaze was only mildly affected. Horizontal gaze to the left could only be maintained very briefly, and there was no impairment to the right. The patient had marked saccadic eye movements in both horizontal planes when following a target (pendulum). He complained of diplopia, particularly in the primary position. Convergence reaction was tested at the bedside by moving a target towards the nose of the patient. It was normal in the right eye and absent on the left. The left eye showed neither pupillary constriction nor adduction upon attempted convergence.

Four weeks later, the patient was able to walk with support, although right sided ataxia persisted. At this time the convergence reaction of the left pupil showed a sluggish reaction on attempted convergence without adduction of the eye. Six weeks later convergence reaction of the left eye returned to normal. There was also improvement of the upward gaze palsy (upward gaze was now possible up to 10 degrees) and the horizontal gaze to the left.

Laboratory investigations including HIV and syphilis serology were negative. CT scan showed a haemorrhage in the right pulvinar thalamus descending on the right side into the lamina quadrigemina. Blood was also noted in the fourth ventricle and the lateral ventricles (fig A). Five further CT scans were performed; the development of a hydrocephalus internus due to compression of the Sylvian aqueduct was noted but improved spontaneously. MRT scan was performed one month after the onset of the haemorrhage; it revealed a small lacunar area ventral to right superior colliculus descending to the mesencephalopontine level (fig B). Angiography demonstrated a vascular malformation as the cause of the haemorrhage. It showed an arteriovenous shunt from the right posterior cerebral artery into the sinus rectus, corresponding with the site of the lesion.

\section{Discussion}

The convergence reaction can be disturbed by a number of neurological disorders, particularly the dorsal midbrain syndrome. ${ }^{6}$ The exact neurophysiology of the convergence reaction, however, has not been completely explained. Our patient, presenting with a reversible unilateral convergence paralysis contralateral to the site of the lesion, raises questions on the physiology of the convergence reaction in regard to fibre crossing in the 
Figure A) Acute CT scan showing the thalamotectal haemorrhage. Blood in third ventricle before the development of

hydrocephalus; B) $M R T$

scan six weeks after the acute onset showing a lacunar lesion in the right pretectal area.
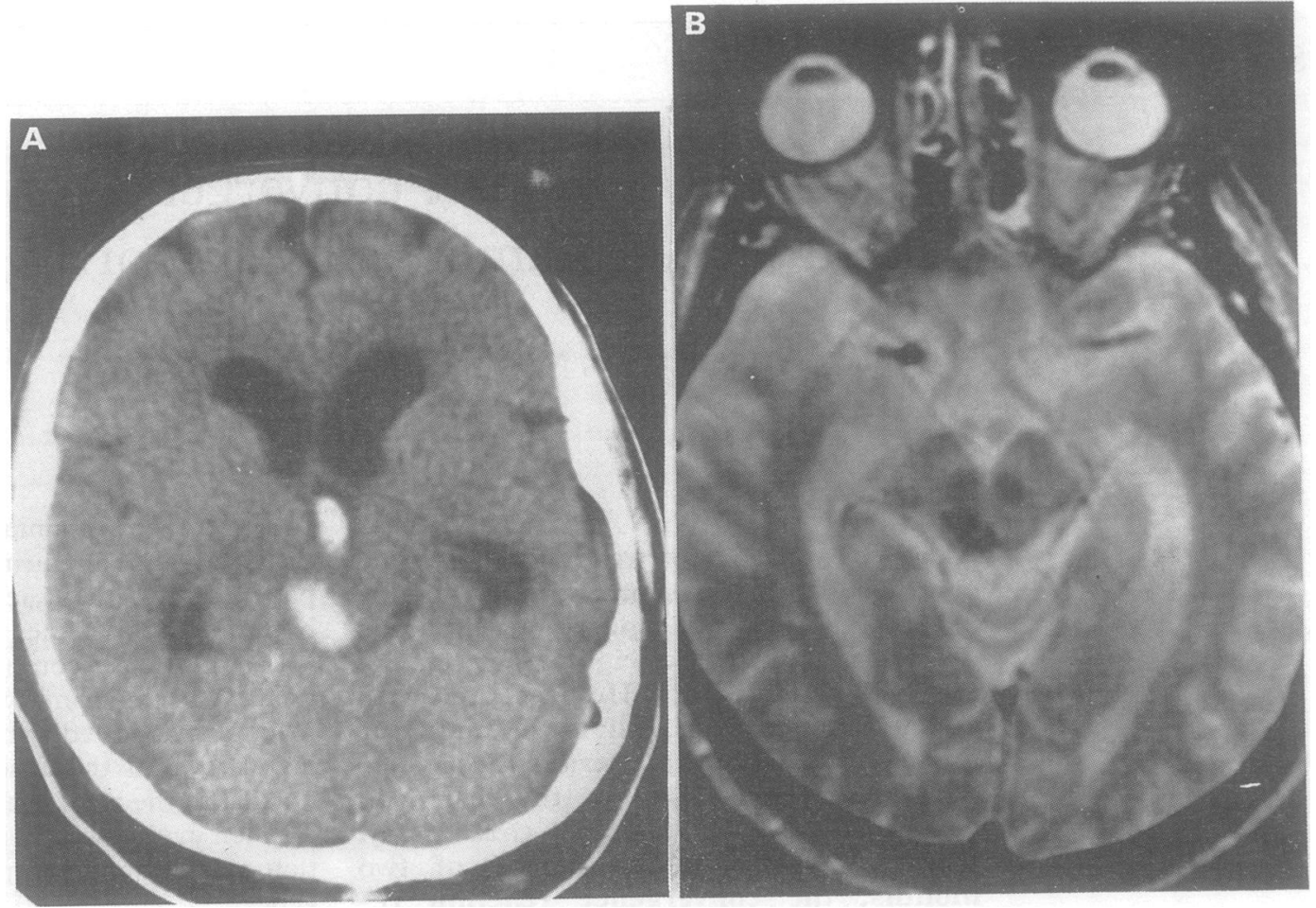

midbrain and the pathways for pupillary light and near reaction.

Previously Warwick's study, ${ }^{7}$ based on primate anatomy and human pathology, challenged Perlia's midbrain centre of convergence reaction. Nevertheless, a rather mechanistic concept of a median convergence centre in the midbrain was maintained. Supported by stimulation experiments, Warwick ${ }^{7}$ assumed a cortical centre of control of convergence, a view confirmed by Jampel's experimental work ${ }^{8}$ and recent observations of convergence impairment associated with supratentorial brain lesion. ${ }^{9}$ In addition to the posterior parietal and the frontal cortex (Area 8), the preoccipital cortex (Area 18, 19, 22) is considered to be the cortical convergence centre. ${ }^{10}$ Retinal blur and binocular disparity serve as input for the accommodation and vergence reactions. Fibres from the cortical convergence areas project via the internal corticotectal tracts to the tectum of the midbrain. ${ }^{11}$ Although some fibres have been traced to the abducens nucleus, ${ }^{8}$ the exact pathways to the oculomotor neurons are not precisely known. A predominantly homolateral projection is assumed. ${ }^{8}$ It has been shown by experiments, that unilateral cortical stimulation produced adduction of the homolateral eye, although unequal adduction was also noted. ${ }^{8}$

The clinical findings in our patient challenge the concept of the homolateral corticotegmentooculomotor projection and suggest that descending fibres from the supratentorial convergence areas have a complete or partial fibre decussation in the thalamotectal area before reaching their last neuron in the oculomotor nucleus. The reversibility of the unilateral convergence paralysis contralateral to the thalamotectal lesion may either suggest a transient block by local oedema due to the haemorrhage or a functional recovery due to the efficacy of the contralateral fibres descending into the midbrain.

Distinct pathways for pupillary light and pupillary convergence reaction have been sugand near reaction of the left eye supports this view. The undisturbed pupillary light reaction of the left eye rules out an incomplete oculomotor palsy.

Our case of a transient unilateral convergence paralysis contralatral to the thalamotectal lesion, suggests a cortical convergence centre with fibre crossing to efferent neurons of Furthermore, the independent pathways for light and convergence are confirmed by our findings. Although a bilateral representation of the cortical near reflex has been demonstrated in stimulation experiments, ${ }^{8}$ the projection systems into the tectum and midbrain have been identified and different pathways for convergence and light reaction have been established. It is still unclear to what extent fibre decussation occurs in the midbrain, and the exact localisation of unilateral convergence impairment remains unknown.

1 Leigh RJ, Zee DS. The neurology of eye movements. Philadelphia: FA Davies Company. 1983:125-40.

Parinaud $H$. Paralysis of the movement of convergence of the eyes. Brain 1886:9:330-41.

the eyes. Brain 1886:9:330-41. Stuttgart, Berlin, Köln
Ludin HP. Das Parkinsonsyndrom. Stutg Mainz: Kohlhammer, 1988:59.

4 Walsh FB, Hoyt WF. Clinical Neuro-ophtalmology. (3rd ed) Baltimore: Williams \& Wilkins Company, 1969:1007-8. Adams RD, Victor M. Principles of neurology. (4th ed) New York, St. Louis, San Francisco: McGraw-Hill, New York,

6 Keane JR. The pretectal syndrome: 206 patients. Neurology 1990;40:684-90.

7 Warwick $R$. The so called nucleus of convergence. Brain 1955;78:92-114.

8 Jampel RS. Presentation of the near response on the cerebral cortex of the macaque. Am $\mathcal{F}$ Ophtalmol 1959;48. 573-82. gested. ${ }^{81213}$ Our patient's dissociation of light the oculmotor nerve in the tectal region. 
9 Ohtsuka K, Maekawa H, Takeda M, Uede N, Chiba S Accommodation and convergence insufficiency with left middle artery occlusion. Am $f$ Ophtalmol 1988;106: $60-4$

10 Judge SJ, Cumming G. Neurons in the monkey midbrain with activity related to vergence eye movement and accommodation. F Neurophysiol 1986;55:915-30.

11 Crosby EC, Henderson JW. The mammalian midbrain and isthmus regions; fiber connections of the superior collicu- lus, pathways concerned in automatic eye movements. $\mathcal{f}$ Comp Neurol 1948;88:53-91.

12 Gamlin PD, Gnadt JW, Mays LE. Abducens internuclear neurons carry an inappropriate signal for ocular convergence. I Neurophysiol 1989;62:70-81.

13 Gamlin PD, Gnadt JW, Mays LE. Lidocain induced unilateral internuclear ophtalmoplegia. Effects on convergence and conjugate eye movements. F Neurophysiol 1989;62:82-95. 